\title{
The interaction between serotonin transporter allelic variation and maternal care modulates sociability on Instagram
}

\author{
Andrea Bonassi ${ }^{\mathrm{a}, \mathrm{b}}$, Ilaria Cataldo ${ }^{\mathrm{a}}$, Giulio Gabrielic ${ }^{\mathrm{c}}$, Moses Tandiono ${ }^{\mathrm{d}, \mathrm{e}}$, Jia \\ Nee Foo ${ }^{\mathrm{d}, \mathrm{e}}$, Bruno Lepri $^{\mathrm{b}}$, Gianluca Esposito ${ }^{\mathrm{a}, \mathrm{c}, \mathrm{d}, *}$ \\ ${ }^{a}$ Department of Psychology and Cognitive Science, University of Trento, Rovereto, Italy \\ ${ }^{b}$ Mobile and Social Computing Lab, Foundation Bruno Kessler, Trento, Italy \\ ${ }^{c}$ Psychology Program, School of Social Sciences, Nanyang Technological University, \\ Singapore, Singapore \\ ${ }^{d}$ Lee Kong Chian School of Medicine, Nanyang Technological University, Singapore, \\ Singapore \\ ${ }^{e}$ Human Genetics, Genome Institute of Singapore, Singapore, Singapore
}

\begin{abstract}
In both physical and online environments, human social interactions can represent a means to gain recognition and approval from others. While there is considerable evidence about the interaction between genetics and the environment on social development, studies adopting the same framework to investigate online social interactions are lacking. The present work applies a model from behavioural genetics on Instagram sociability to explore the impact of individual development on social media platforms' behaviour.

We hypothesized that social attitudes on Instagram resulted from an interaction between serotonin transporter gene alleles and the individual's remembrance of the interaction with caregivers. We assessed the environmental and genetic components of 57 Instagram users, adopting the Parental Bonding Instrument to determine the perceived parental care and overprotection. The number of posts followed by users ("followings") and followers were collected from participants' Instagram profiles as online social activity indexes. Additionally, the ratio between the number of followers and followings ("Social
\end{abstract}

\footnotetext{
*Corresponding author

Email address: gianluca.esposito@ntu.edu.sg I gianluca.esposito@unitn.it (Gianluca Esposito)
} 
Desirability Index") was calculated to estimate each user's social network's asymmetry. Finally, information on the polymorphism rs25531 (T/T homozygotes vs C-carriers) within the serotonin transporter gene was extracted from participants' buccal mucosa cell samples.

In line with the predictions, we found a gene-environment interaction on the "Social Desirability Index": users with the genotype more sensitive to environmental influences ( $\mathrm{T} / \mathrm{T}$ homozygotes) showed a higher Instagram "Social Desirability Index" than less sensitive ones (C-carriers) when positive maternal care was experienced.

This result may contribute to the understanding of online social behaviour from a gene*environment perspective.

Keywords: Gene * Environment, Parental Bonding, Maternal Care, Serotonin Transporter Gene, rs25531, Instagram, Social Media, Social Network Sites, Online Behavior, Instagram

\section{Introduction}

Among the early environmental factors, parent-infant interaction modulates human development on a biological level, influencing social, emotional and cognitive domains [1]. Efficient parenting promotes an inclusive environment, fostering patterns for high-quality social relationships in childhood $[2,3]$ which, in turn, can decrease behavioral and physiological distress in adulthood [4]. Simultaneously, different long-term environmental factors can play an active role in the modulation of sociality across the human lifespan. From the first years of their lives, children are increasingly exposed to technological devices [5], and begin to use mobile technologies before school age [6]. This may contribute to social competencies learning in both online and offline worlds [7]. Increasingly, younger and younger individuals - as age limits can be easily deceived - can access social network sites (SNSs), where social exchanges between users are immediate, free, continuous and pervasive [8]. Among these sites, Instagram (IG) is a photo- and video-sharing platform where users can follow stories, publish or comment on posts, watch or upload content, and chat privately. Some evidence demonstrated that increased Instagram activity might be associated with positive outcomes, acting as a motivator for a satisfying self-presentation and increasing self-confidence [9], but it is also associated with negative consequences, like a risk to the development of a depressed mood [10] and a detrimental effect on mental well-being 
[11].

However, the influence of environmental cues might not fully explain such a tangled phenomenon, such as social behaviour. According to the biopsychosocial model of wellness and illness, it is more likely that genetic predispositions could be crucial in shaping the effect of early experiences on social conduct on SNSs. At the molecular level, individual behaviours result from genetic predispositions and environmental stressors or conditions, especially during critical periods [12]. According to the differential susceptibility model [13] a given genetic predisposition is not uniquely "good" or "bad" in human behaviour. Indeed, the level of susceptibility to life events is flexible and regulated by the allelic expression involved in the physiological and behavioural responses to a range of triggering environmental hazards. In the myriad of biological regulators, serotonin is a neurotransmitter implicated in the neural circuits of emotional regulation, social functioning and social affiliation [14, 15]. Serotonin level may vary across brain regions and may have different effects on individuals [16]. The serotonin transporter encoded by the serotonin transporter gene (SLC6A4) is the main regulator of the serotonin removal from the synaptic clefts. Specifically, the promoter region of the serotonin transporter gene (5-HTTLPR) and the adjacent upstream polymorphism (5-HTT rs25531) $[17,18]$ have been investigated in association with perceived attachment and parental attitudes [19, 20, 21]. Concerning 5-HTTLPR, for instance, Truzzi et al. [22] discovered that men with a higher predisposition (carriers of the short form of 5-HTTLPR) for sensitivity to maternal overprotection showed distressed heart rate to female cry. Concerning rs25531, two allelic forms have been found: the substitution of thymine $(\mathrm{T})$ and cytosine $(\mathrm{C})$, with the paired nucleotides guanine $(\mathrm{G})$ and adenine $(\mathrm{A})$, shows differential susceptibility to stressors [23, 24, 25]. However, until now, literature has not been able to disentangle which variation is more associated with adaptive social responses $[26,27]$. Genetic vulnerability conferred by the $\mathrm{A} / \mathrm{A}$ and $\mathrm{T} / \mathrm{T}$ genotype, whose transcription processes are associated with a lower re-uptake of serotonin, can be moderated by social support of parents [28]. The degree of social support received in childhood rules the risk towards mental disorders in adulthood and facilitated a wide range of possible behavioural outcomes [24, 29]. For instance, people with a history of lowquality parental support in childhood could show maladaptive behaviours, and a greater benefit from others' support in adulthood [30, 31, 32, 33]. Conversely, individuals with the $\mathrm{C}$ or $\mathrm{G}$ allele showed enhanced serotonin degrees combined with lessened sensitivity to pain, traumatic events, and 
care [25]. Within the social context, higher social avoidance and lower emotional engagement were related to a decrease of serotonin, whereas higher prosocial behaviour and lower social anxiety from childhood were related to an increase in serotonin $[20,24]$.

In summary, we could assert that sociability emerges from the combined effects of nature, driven by genetic and biological predispositions, and nurture (e.g., social environment, social education across development, social experiences with parents and peers) [12]. However, the function of the "sociability genes" (i.e. 5-HTT rs25531) according to the early environment's quality on the online social interaction of adults is not yet investigated.

Therefore, this research explores how the serotonin transporter gene and the individual's relationship with caregivers during childhood interact in modulating adults' online social interactions on Instagram. Our attention focused on Instagram, one of the most popular social networks, to unveil online social interaction's fundamental mechanisms. Instagram users were firstly required to fill the Parental Bonding Instrument as a measure of their recalled parental bonding. Buccal mucosa samples were subsequently collected and one 5-HTT single nucleotide polymorphism (SNP: rs25531) was examined as a genetic factor. Finally, three social media indexes were assessed from the Instagram profile of each user: the number of (i) published posts as an index of the social productivity of the network, (ii) number of followed users (here called "followings") as a measure of prosocial activity, (iii) number of followers as a measure of the social attraction of other users. A fourth Instagram index was obtained as a proxy of asymmetry of the social network, calculated through the ratio between the number of followers and the number followings: (iv) the "Social Desirability Index" (SDI).

For each Instagram index as the dependent variable, we hypothesized an interaction effect between the genetic component and the attachment scores, independent of the gender. More precisely, we expected that adult Instagram users with a genetic risk factor (rs25531 T/T) who experienced a supportive and positive past relationship with parents (High Parental Care, Low Parental Overprotection) would show a greater online social activity compared to protective genetic carriers (rs25531 C-carriers). Although we formulated a directional hypothesis, we could not predict a-priori on which Instagram variable potential effects would have been displayed. Given the new role of Instagram activity under a gene-environment perspective, we adopted an exploratory approach. 


\section{Methods}

\section{Participants}

Sixty-one $(N=61)$ young adults participated among the students of the Nanyang Technological University (Singapore). Inclusion criteria were: (i) no history of genetic, neurological or psychiatric disorders, (ii) age lower than 30 years old, and (iii) owning and using an Instagram account. Four participants were omitted due to technical issues; thus our final sample included 57 Singaporean non-parent adults (16 males and 41 females) aged 18-25 years old $(M=20,82, S D=1,59)$.

\section{Procedure}

Before collecting data, participants provided informed consent and reported demographic details. The study included three assessments: 1) participants completed the self-report questionnaire Parental Bonding Instrument on the web-based survey platform Qualtrics and 2) supplied the link of their Instagram page from which a Python program extracted four parameters of their on-line activity. When the Python program failed, Instagram data was assessed manually. Finally, 3) a sample of buccal mucosa was collected from each participant and genotyped in the laboratory.

\section{Parental Bonding}

The Parental Bonding Instrument (PBI) [34] is a 50-item self-report questionnaire administered to adults to quantify their parental bonding during childhood and adolescence. This instrument allows participants to recollect the quality of the relationship with their parents, rating their caregiving behaviour on a 0-3 Likert scale. The PBI (averaged Cronbach's $\alpha=0.88$ in our sample) provides an estimate of the reported early attachment across four subscales: maternal and parental care, maternal and parental overprotection (see Supplementary Materials). While the two dimensions of care evaluate the level of parental closeness and affection (e.g. "Could make me feel better when I was upset"), the two subscales of overprotection measure the level of parental imposition or detachment (e.g. "Made me feel I wasn't wanted"). The PBI is an instrument widely used worldwide with reliable psychometric properties in terms of high validity, internal consistency [35, 36], and previously used in gene*environment research paradigms [37, 22, 38]. 


\section{Instagram Parameters}

We selected four variables from Instagram data: number of posts, number of followings, number of followers, and Social Desirability Index. The number of posts describes the quantity of published and shared material on the participant's profile. On Instagram, posts can usually be pictures, videos or graphical texts, all content that other users can actively comment on and like [39]. This parameter suggests the users' level of productivity and desire for exposure to others' judgements. The number of posts was also associated with the users' psychological vulnerability (i.e. depression) [40]. The number of followings represents the amount of followed users by an assessed study participant. Instagram people compare their behaviour with other users who become the reference points of their virtual activity. More followings demand more social interactions in terms of greater time invested in online social activities and higher passive exposure to content published by other users [41, 42]. The number of followers is the number of users who follow the assessed study participant. Followers' recognition and support act as a reward for the user's online behaviour, who then adjusts his/her social activity in line with the social network's requests to boost further agreeableness (i.e. the number of likes) [43]. The Social Desirability Index is obtained by the ratio between the number of followers and the number of followings. We calculated this ratio to explore the asymmetry between these two parameters in each participant's network. As discussed in a previous work [38], this parameter has the potential to reveal "the tendency of some Instagram users of maximizing the number of followers at the expense of the number of followings".

\section{Genotyping}

The same DNA sequencing technique adopted by Bonassi and colleagues [37] was used in this study. DNA extraction and genotyping were performed by ACGT, Inc. (Wheeling, IL) for the 5-HTT gene (SLC6A4) rs25531 region target. DNA was derived from each kit, applying the Oragene DNA purifying reagent. DNA concentrations were evaluated through spectroscopy (NanoDrop Technologies, USA). A polymerase chain reaction (PCR) reaction of $20 \mathrm{ll}$ including $1.5 \mathrm{ll}$ of genomic DNA from the test sample, PCR buffer, $1 \mathrm{mM}$ of each primer (forward 5'-GGCGTTGCCGCTCTGAATGC-3' and reverse 5'-GAGGGACTGAGCTGGACAACCAC-3') $10 \mathrm{mM}$ deoxyribonucleotides, KapaTaq polymerase, and $50 \mathrm{mM} \mathrm{MgCl} 2$ was administered. PCR operation consisted of first a 15 minute denaturation at $95{ }^{\circ} \mathrm{C}$, and 35 cycles 
at $94{ }^{\circ} \mathrm{C}(30 \mathrm{~s}), 60{ }^{\circ} \mathrm{C}(60 \mathrm{~s}), 72^{\circ} \mathrm{C}(60 \mathrm{~s})$ and a further 10 minute lengthening at $72{ }^{\circ} \mathrm{C}$. PCR reactions were genotyped with an ABI 3730xl Genetic Analyzer (Applied Biosystems Inc.) and standardized with GeneScan 600 LIZ (Applied Biosystems, Inc.) size standards on each sample. Genotypic data were analyzed by GeneMapper ID (Applied Biosystems, Inc.).

The average distribution of the genotypes in the Asiatic population is $86-87 \%$ for $\mathrm{T} / \mathrm{T}$ homozygotes and $13-14 \%$ for $\mathrm{C}_{\text {-carriers }}{ }^{1}$, whereas the distribution in our sample was $73.68 \%$ for $\mathrm{T} / \mathrm{T}$ homozygous $(\mathrm{N}=42), 26.32 \%$ for $\mathrm{C} / \mathrm{T}$ heterozygous $(\mathrm{N}=15)$ and $0 \%$ for $\mathrm{C} / \mathrm{C}$ homozygous $(\mathrm{N}=0)$. This genotype distribution follows the Hardy-Weinberg Equilibrium $\left(X^{2}(1)=1.31\right.$, ns). Participants having one $\mathrm{C}$ allele $(\mathrm{C} / \mathrm{T})$ were classified into a single $\mathrm{C}$-carriers group. Participant gender $\left(X^{2}(1)=0.23\right.$, ns $)$ did not significantly differ between the two groups $\mathrm{T} / \mathrm{T}$ vs $\mathrm{C}$.

\section{Analysis}

Data were analyzed on $\mathrm{R}$ (version 4.0.0). Instagram variables were first standardized with z-scores (Table 1). Measured variable distributions were checked for normality, skewness, kurtosis, presence of outliers and were then visualized by density and quantile-quantile plots (Table 1 ).

The distance of each observation to the centroid was estimated to detect outliers defined with a value equal to $2 S D s$ above/below the mean [44]. Three extreme values were identified as outliers for the number of followings and the number of posts, two values for the SDI, and one value for the number of followers out of total 57 observations for each Instagram variable. They were replaced with the mean value in observations, obtained by excluding the outliers. Each Instagram variable showed a normal univariate distribution. Only the number of posts did not present a Gaussian distribution, and a logarithmic transformation was applied to allow for the adoption of parametric tests. Moreover, the assumptions of homogeneity of variance and multicollinearity across the sample was verified (see Supplementary Materials).

A preliminary analysis was executed on Instagram variables to exclude any effect of participants' gender (4 repeated measures, $\alpha=0.0125$ ). An equivalent statistical procedure was adopted for Instagram variables overall for

\footnotetext{
${ }^{1} 1000$ Genomes project, BioSample: SAMN07486024, dbSNP (Short Genetic Variations), 2017
} 


\begin{tabular}{lcccccc}
\hline \multicolumn{7}{c}{ Instagram Variables } \\
\hline Variable & Min & 1st Q & Median & Mean & 3rd Q & Max \\
Posts Number & 0.33 & 0.39 & 0.49 & 0.56 & 0.67 & 1.19 \\
Followings Number & -1.54 & -0.77 & -0.12 & -0.12 & 0.52 & 1.80 \\
Followers Number & -0.80 & -0.50 & -0.16 & -0.11 & 0.22 & 1.45 \\
Social Desirability Index & -1.16 & -0.28 & -0.13 & -0.16 & -0.03 & 0.61 \\
\hline \multicolumn{7}{c}{ Parental Bonding Dimensions } \\
\hline Variable & Min & 1st Q & Median & Mean & 3rd Q & Max \\
Maternal care & 0.00 & 4.00 & 8.00 & 9.97 & 14.00 & 28.00 \\
Paternal care & 0.00 & 9.00 & 14.00 & 14.49 & 18.00 & 30.00 \\
Maternal Overprotection & 8.00 & 18.00 & 23.00 & 23.82 & 30.00 & 37.00 \\
Paternal Overprotection & 11.00 & 23.00 & 29.00 & 27.05 & 32.00 & 37.00 \\
\hline
\end{tabular}

Table 1: Descriptive statistical summary of each variable: minimum (Min), first quartile (1st Q), median, mean, third quartile (3rd Q) and maximum (Max).

our hypothesis-driven analysis. Four multiple linear regressions were performed with the Instagram value as the dependent variable, the 5-HTT gene genotype rs25531 ( $\mathrm{T} / \mathrm{T}$ and $\mathrm{C}$ carriers) as a between-subjects factor and the PBI dimensions (i.e. maternal care, maternal overprotection, paternal care, and paternal overprotection) as continuous covariates. Hence, a Bonferroni correction was applied for the number of tests (4 repeated measures, $\alpha=0.0125)$. Post-hoc power analysis was calculated with $G^{*}$ Power software (version 3.1) [45]. One 2-way interaction effect was obtained and represented by a barplot and a scatterplot with linear models. Pearson's $r$ and Fisher's $z$ coefficients were used to estimate the effect of the covariate on the dependent variable, while $R^{2}$ and Cohen's $d$ were calculated to quantify the size of the significant effects.

For the significant interaction between the genotype and the PBI covariate on the Instagram variable, post-hoc Student's $t$-tests (2 repeated measures, $\alpha=0.025$ ) were performed within the low vs high PBI groups (obtained by the median split procedure) to prove differences between the two genetic clusters.

\section{Results}

Means and standard deviations of the measured variables are reported in Table 2 . 


\begin{tabular}{lcccc}
\hline \multicolumn{5}{c}{ Number of Posts } \\
\hline PBI Dimension & Low/TT & Low/C & High/TT & High/C \\
Maternal Care & $0.57(0.05)$ & $0.56(0.07)$ & $0.55(0.05)$ & $0.57(0.12)$ \\
Paternal Care & $0.56(0.05)$ & $0.50(0.04)$ & $0.57(0.05)$ & $0.64(0.12)$ \\
Maternal Overprotection & $0.57(0.05)$ & $0.63(0.12)$ & $0.56(0.04)$ & $0.50(0.04)$ \\
Paternal Overprotection & $0.57(0.05)$ & $0.65(0.12)$ & $0.55(0.05)$ & $0.51(0.06)$ \\
\hline \multicolumn{5}{c}{ Number of Followings } \\
\hline PBI Dimension & Low/TT & Low/C & High/TT & High/C \\
Maternal Care & $0.11(0.19)$ & $-0.27(0.21)$ & $-0.27(0.17)$ & $0.01(0.75)$ \\
Paternal Care & $-0.19(0.17)$ & $-0.60(0.18)$ & $0.01(0.20)$ & $0.28(0.41)$ \\
Maternal Overprotection & $-0.09(0.19)$ & $-0.034(0.39)$ & $-0.11(0.18)$ & $-0.33(0.30)$ \\
Paternal Overprotection & $-0.12(0.16)$ & $0.32(0.34)$ & $-0.06(0.22)$ & $-0.53(0.28)$ \\
\hline & Number of Followers & & \\
\hline PBI Dimension & Low/TT & Low/C & High/TT & High/C \\
Maternal Care & $-0.03(0.13)$ & $-0.19(0.13)$ & $-0.15(0.10)$ & $-0.10(0.38)$ \\
Paternal Care & $-0.12(0.11)$ & $-0.29(0.17)$ & $-0.07(0.11)$ & $-0.02(0.20)$ \\
Maternal Overprotection & $-0.07(0.12)$ & $-0.15(0.20)$ & $-0.13(0.10)$ & $-0.17(0.19)$ \\
Paternal Overprotection & $-0.10(0.11)$ & $0.16(0.22)$ & $-0.09(0.12)$ & $-0.38(0.13)$ \\
\hline & Social Desirability Index & & \\
\hline PBI Dimension & Low/TT & Low/C & High/TT & High/C \\
Maternal Care & $-0.27(0.08)$ & $-0.19(0.04)$ & $-0.02(0.05)$ & $-0.34(0.18)$ \\
Paternal Care & $-0.21(0.07)$ & $-0.23(0.08)$ & $-0.05(0.06)$ & $-0.23(0.08)$ \\
Maternal Overprotection & $-0.11(0.08)$ & $-0.22(0.09)$ & $-0.16(0.06)$ & $-0.23(0.07)$ \\
Paternal Overprotection & $-0.14(0.07)$ & $-0.17(0.06)$ & $-0.13(0.06)$ & $-0.26(0.08)$ \\
\hline
\end{tabular}

Table 2: Mean values in T/T homozygotes and C-carriers divided by PBI dimensions (high or low) on Instagram variables. Standard error means are reported between parentheses.

\section{Instagram variables: Preliminary Results}

Four two-tailed Student's $t$-tests were conducted (see Table 3) to exclude any influence of gender on Instagram variables. Only the number of followings was found to be higher in males than females $(t=2.60$, df $=55$, $p<0.0125)$. Consequently, participant gender was included as a betweensubjects variable limited to the number of Instagram followings.

\section{Instagram Interaction Effects}

Social Desirability Index. Results of the regression analysis on the standardized SDI $\left(F(9,47)=1.92, R^{2}=0.27\right.$, power $\left.=0.83\right)$ revealed a significant interaction between maternal care and genotype emerged for Instagram SDI 


\begin{tabular}{lcc}
\hline Instagram Variable & Males & Females \\
\hline Posts Number & $0.60(0.05)$ & $0.55(0.03)$ \\
Followings Number & $0.32(0.23)$ & $-0.30(0.12)$ \\
Followers Number & $0.08(0.12)$ & $-0.19(0.08)$ \\
Social Desirability Index & $-0.11(0.03)$ & $-0.18(0.05)$ \\
\hline
\end{tabular}

Table 3: Mean values in male and female participants on Instagram variables. Standard error means are reported between parentheses.

$(\beta=0.04, \mathrm{SE}=0.01, t=2.86, p<0.006)$. The distribution of genotypes TT vs C-carriers was not significantly different between high vs low maternal care $\left(X^{2}(1)=2.46\right.$, ns). No main effect of covariate, genotype or other interactions with genotype were significant. Maternal care was negatively associated with the Instagram SDI for C-carriers $(t(15)=-2.50, \mathrm{df}=13, r$ $=-0.57, p<0.03)$, but positively associated with the Instagram SDI for $\mathrm{T} / \mathrm{T}$ homozygous $(t(42)=2.75, \mathrm{df}=40, r=0.40, p<0.01)$ (Figure 1$)$. A significant difference between the slopes for $\mathrm{T} / \mathrm{T}$ and $\mathrm{C}$-carriers was confirmed by Fisher's $z(z=3.24, p<0.001)$. Moreover, one-tailed post-hoc Student's $t$ tests on the $\mathrm{C}$-carriers vs $\mathrm{T} / \mathrm{T}$ in low and high maternal care groups revealed that the SDI was significantly different between $\mathrm{C}$-carriers and $\mathrm{T} / \mathrm{T}$ homozygotes only when they reported past experiences of high maternal care $(t=$ $2.30, \mathrm{df}=25, p<0.025)$, but not when they had a past of low maternal care $(t=-0.76, \mathrm{df}=28, \mathrm{~ns})$ (Figure 1$)$. Homogeneity of variance of the SDI by maternal care was checked $\left(K^{2}=0.12\right.$, df $=1$, ns $)$.

Number of Instagram Posts, Followings and Followers. No significant main effect or interactions were found.

\section{Discussion}

In this study, we probed how alleles in 5-HTT rs25531 interact with recalled parental care and overprotection in the modulation of social behaviour on Instagram. Specifically, we hypothesized one genotype*environment interaction (5-HTT rs25531 SNP * parental bonding in childhood) on Instagram variables.

In accordance with the hypothesis, we discovered that adult Instagram users with genetic vulnerability ( $\mathrm{T} / \mathrm{T}$ homozygotes) showed varying Instagram social conduct related to the quality of the parental practices experienced in childhood. As expected, T/T homozygotes who reported high maternal care 


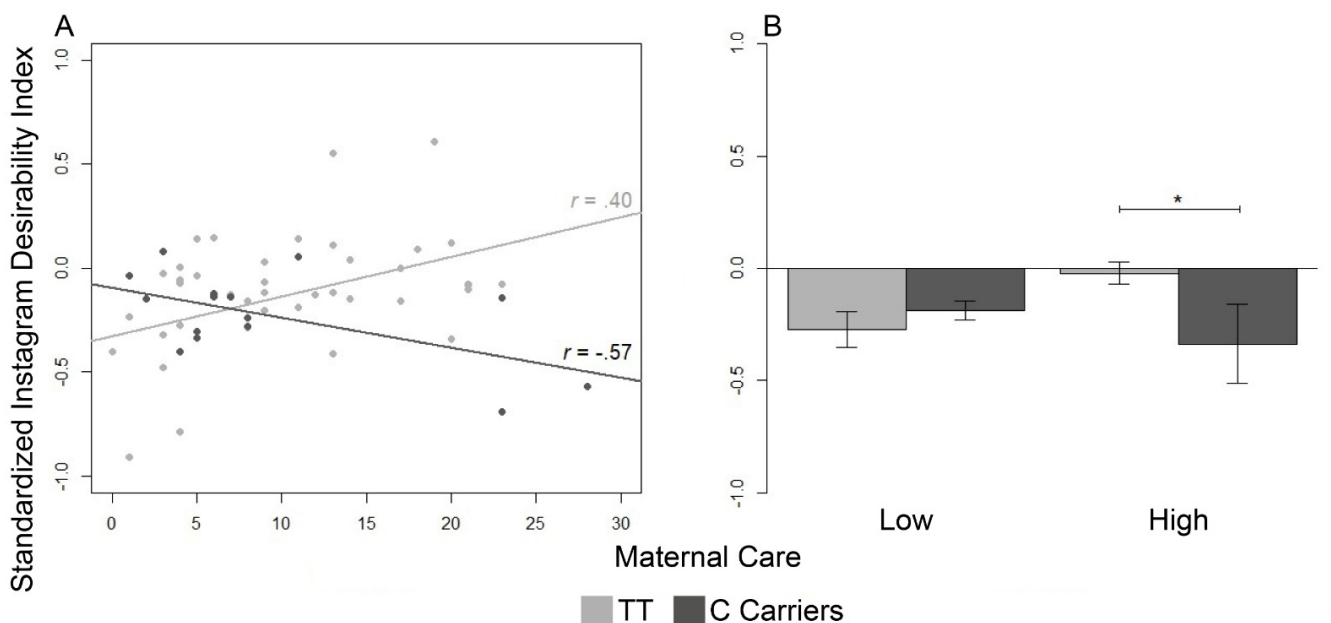

Figure 1: A: Effect of the interaction between genotype and maternal care on the standardized Instagram Social Desirability Index. Correlations between the Social Desirability Index and the reported maternal care. Black circles $=\mathrm{C}$-carriers; grey circles $=\mathrm{T} / \mathrm{T}$ homozygotes. Lines represent the linear models for C-carriers (black) and $\mathrm{T} / \mathrm{T}$ homozygotes (grey). Pearson's $r$ values are reported. B: Comparison between Social Desirability Index in C-carriers (black) and $\mathrm{T} / \mathrm{T}$ homozygotes (grey) divided into high and low maternal care. $(* p<0.025)$

scores exhibited an increasing trend in the Instagram SDI. In contrast, those who reported low scores in the equivalent dimension determined a decreasing trend in the same index. Interestingly, C-carriers showed the opposite pattern: as maternal care scores were lower, Instagram SDI increased. In particular, vulnerable genetic carriers ( $\mathrm{T} / \mathrm{T}$ homozygotes) with a positive relationship with the mother displayed a higher Instagram SDI than protective genetic carriers (C-carriers).

Our findings were consistent with previous results on 5-HTT and rs25531, which suggested a large variability between carriers in the behavioural response to social and distressing stimuli [46, 47, 22]. Elucidating the moderating role of the genotype on the sensitivity of the phenotype to environmental influences [24], the differential susceptibility model states that an individual can be more or less genetically susceptible to the beneficial versus dysfunctional effect generated respectively by enriching or undermining circumstances $[13,12]$. This study investigated the present model on rs25531 within a novel and innovative framework: the online social behaviour on Instagram, an SNS. In line with the model [13], a T versus C allele's pres- 
ence implicated a conditional variation in the online social response: $\mathrm{T} / \mathrm{T}$ homozygotes who benefited from efficacious maternal care displayed a higher Instagram SDI than C-carriers. In this condition, Instagram users carrying the $\mathrm{C}$ allele were shown to be less susceptible than $\mathrm{T} / \mathrm{T}$ homozygotes to supportive maternal care during childhood. Compared to $\mathrm{T} / \mathrm{T}$ genotype, the $\mathrm{C}$ allele conferred lower vulnerability to adverse conditions linked to neglecting caregiving and less advantage from a favourable and positive relationship with the mother. As a consequence, users with the $\mathrm{C}$ allele exhibited an Instagram social response unconcerned about the advantages promoted by an appropriate maternal caring $[48,25]$. Otherwise, users with the T/T genotype took advantage of a protective and comfortable maternal relationship, exhibiting higher asymmetry between the number of followers and followings than less sensitive ones [49]. As a result of developmental flexibility, the T allele predisposes individuals to be more vulnerable or plastic according to the functional or dysfunctional attachment with the mother [50, 51, 52].

The present research extends previous evidence of genetic influence on the frequency of online social behaviour $[53,54,55]$. Regarding 5-HTT rs25531, a previous study found that carriers of susceptibility genetic factors $(\mathrm{T} / \mathrm{T}$ homozygotes) presented a higher Instagram number of followings than nonsensitive ones (C-carriers) when they reported a high level of confidence toward others [56]. As pointed out by another work [38], in the current study, users genetically susceptible to environmental influences displayed higher SDI when they have previously experienced positive maternal bonding.

We focused our attention on the SDI because it represents the distribution of contacts for each user on Instagram. This index attributes a size to the structure of each user's network, where the number of followers increased at the cost of the number of followings. Maximizing the SDI means enlarging the network structure to achieve greater approval and benefit from the masses $[57,58]$. Within this frame, we argue that higher maternal care could determine the basis of new prosocial behaviour in the offline environment, later combined with a greater need for social desirability in the online environment [59, 9, 38]. An Instagram user sensitive to early experiences with the mother could, in turn, respond more efficaciously to the request of others and support social exchanges in response to their followers' expectations $[60,61,43]$. 


\section{Conclusion}

This research is not exempt from some limitations. Firstly, the low sample size was constrained by data collection of multiple variables in a fixed period. Moreover, data collection was circumscribed to young adults, and the participants' socioeconomic status (SES) was not evaluated. For instance, users who live in a situation of economic security could increase their Instagram activity to prove their ideal prosperity to the followers [62]. The year of the first registration on Instagram was also not collected; thus, the indexes' quantities were not controlled for the duration of online activity. Furthermore, the analysis was performed on no more than three Instagram parameters and one combined index that did not factor in "bot accounts" or "artificial" followers that can be purchased instead of being earned through social capital $[63,64]$. Finally, the recalled parental bonding was measured with a self-reported instrument.

Future studies should consider a more extensive and multicultural sample to probe potential cross-cultural differences in Instagram behaviour and a gender-balanced sample that includes participants of different ages. The assessment of plausible moderators of Instagram usage like the SES and the sense of membership with peer groups may also be considered. The increase in the number of posts, followers and followings should be traced in relation to the year of the first registration on Instagram. Novel Instagram variables and indexes able to detect the variety of the shared content may also be taken into account, such as Instagram number of stories and tagged posts or the time spent using Instagram actively (i.e., chatting, posting) and passively (i.e., viewing, scrolling). To detect the long-term impact of early life, a longitudinal study to analyze the development of online social skills as an effect of genes and experiences is recommended. Furthermore, physiological measures from peripheral and central nervous systems could be adopted to discover potential differences between online and offline social responses. Finally, as rs25531 and 5-HTTLPR result in two independent loci for analysis [18], this model could be implemented on 5-HTTLPR.

In conclusion, serotonin transporter gene polymorphisms (rs25531) and early caregiving behaviours play an interactive role in the development of online social behavior in Instagram users. Combined with previous evidence, these results offer an active contribution to identifying an online social marker in the interplay between genetic and environmental components. 


\section{Ethics}

The research was authorized by the Ethical Committee of Nanyang Technological University (IRB-2015-08-020-01). Informed consent was acquired from all participants, and the study was conducted following the Declaration of Helsinki. Questionnaires' data and Instagram data were anonymized at the beginning of the data collection. The genetic assessment was performed on anonymized biosamples at the Nanyang Technological University (Singapore).

\section{Data Access}

The data of this study can be found in the NTU's Data repository (DRNTU Data) at the following address:

https://doi.org/10.21979/N9/XIVRWS

\section{Authors Contribution}

Conceptualization and Experimental Design: IC, GE, BL. Data Collection: IC. Data Curation: AB, GG. Genetic Analysis: MT, JNF. Data Analysis, Data Interpretation: AB. Writing: AB, IC. Revision: AB, BL, IC, GG, GE. Supervision: GE, BL.

\section{Funding Information}

This research was supported by grants from the NAP SUG to GE (M4081597, 2015-2021).

\section{Acknowledgements}

All participants in this study are gratefully acknowledged. We would also like to acknowledge Dr. Anna Truzzi (Trinity College, Dublin) for the useful suggestions and Lim Mengyu (Nanyang Technological University, Singapore) and An An Lieu (Nanyang Technological University, Singapore) for editing the manuscript.

\section{Conflict of Interest}

The authors declare that the research was conducted in the absence of any commercial or financial relationships that could be construed as a potential conflict of interest. 


\section{References}

[1] M. H. Bornstein, Handbook of parenting: Volume I: Children and parenting, Psychology Press, 2005.

[2] M. H. Bornstein, D. L. Putnick, C.-S. Hahn, C. S. Tamis-LeMonda, G. Esposito, Stabilities of infant behaviors and maternal responses to them, Infancy 25 (2020) 226-245.

[3] C. M. Stocker, A. S. Masarik, K. F. Widaman, B. T. Reeb, J. D. Boardman, A. Smolen, T. K. Neppl, K. J. Conger, Parenting and adolescents' psychological adjustment: Longitudinal moderation by adolescents' genetic sensitivity, Development and psychopathology 29 (2017) 1289.

[4] A. Dalsant, A. Truzzi, P. Setoh, G. Esposito, Maternal bonding in childhood moderates autonomic responses to distress stimuli in adult males, Behavioural brain research 292 (2015) 428-431.

[5] S. L. Calvert, Children and digital media, Handbook of child psychology and developmental science (2015) 1-41.

[6] H. K. Kabali, M. M. Irigoyen, R. Nunez-Davis, J. G. Budacki, S. H. Mohanty, K. P. Leister, R. L. Bonner, Exposure and use of mobile media devices by young children, Pediatrics 136 (2015) 1044-1050.

[7] D. Meshi, D. I. Tamir, H. R. Heekeren, The emerging neuroscience of social media, Trends in cognitive sciences 19 (2015) 771-782.

[8] I. Cataldo, B. Lepri, M. J. Y. Neoh, G. Esposito, Social media usage and development of psychiatric disorders in childhood and adolescence: A review, Frontiers in Psychiatry 11 (2020).

[9] A. Meier, S. Schäfer, The positive side of social comparison on social network sites: How envy can drive inspiration on instagram, Cyberpsychology, Behavior, and Social Networking 21 (2018) 411-417.

[10] E. Frison, S. Eggermont, Browsing, posting, and liking on instagram: The reciprocal relationships between different types of instagram use and adolescents' depressed mood, Cyberpsychology, Behavior, and Social Networking 20 (2017) 603-609. 
[11] S. Ponnusamy, M. Iranmanesh, B. Foroughi, S. S. Hyun, Drivers and outcomes of instagram addiction: Psychological well-being as moderator, Computers in Human Behavior 107 (2020) 106294.

[12] G. Esposito, P. Setoh, K. Shinohara, M. H. Bornstein, The development of attachment: Integrating genes, brain, behavior, and environment, Behavioural Brain Research 325 (2017) 87-89. SI: Development of Attachment.

[13] J. Belsky, M. Pluess, Beyond diathesis stress: differential susceptibility to environmental influences., Psychological bulletin 135 (2009) 885.

[14] T. Canli, K.-P. Lesch, Long story short: the serotonin transporter in emotion regulation and social cognition, Nature neuroscience 10 (2007) 1103-1109.

[15] C. G. Beevers, T. T. Wells, A. J. Ellis, J. E. McGeary, Association of the serotonin transporter gene promoter region (5-httlpr) polymorphism with biased attention for emotional stimuli., Journal of Abnormal Psychology 118 (2009) 670.

[16] L. Mohammad-Zadeh, L. Moses, S. Gwaltney-Brant, Serotonin: a review, Journal of veterinary pharmacology and therapeutics 31 (2008) 187-199.

[17] J. B. Kraft, S. L. Slager, P. J. McGrath, S. P. Hamilton, Sequence analysis of the serotonin transporter and associations with antidepressant response, Biological psychiatry 58 (2005) 374-381.

[18] J. Wendland, B. Martin, M. Kruse, K. Lesch, D. Murphy, Simultaneous genotyping of four functional loci of human slc6a4, with a reappraisal of 5-httlpr and rs25531, Molecular psychiatry 11 (2006) 224-226.

[19] J. E. Morgan, C. Hammen, S. S. Lee, Parental serotonin transporter polymorphism (5-httlpr) moderates associations of stress and child behavior with parenting behavior, Journal of Clinical Child \& Adolescent Psychology 47 (2018) S76-S87.

[20] B. Q. Ford, I. B. Mauss, A. S. Troy, A. Smolen, B. Hankin, Emotion regulation moderates the risk associated with the 5 -htt gene and stress in children., Emotion 14 (2014) 930. 
[21] A. Gazor, H. Fang, J. R. Gagne, An exploratory genetic analysis: Associations between parent depression symptoms, child temperament, and the serotonin transporter gene polymorphism (5 httlpr), Journal of Applied Biobehavioral Research 22 (2017) e12099.

[22] A. Truzzi, M. H. Bornstein, V. P. Senese, K. Shinohara, P. Setoh, G. Esposito, Serotonin transporter gene polymorphisms and early parentinfant interactions are related to adult male heart rate response to female crying, Frontiers in physiology 8 (2017) 111.

[23] G. Ferraguti, E. Pascale, M. Lucarelli, Alcohol addiction: a molecular biology perspective, Current medicinal chemistry 22 (2015) 670-684.

[24] M. A. Schiele, C. Ziegler, K. Holitschke, C. Schartner, B. Schmidt, H. Weber, A. Reif, M. Romanos, P. Pauli, P. Zwanzger, et al., Influence of 5-htt variation, childhood trauma and self-efficacy on anxiety traits: a gene-environment-coping interaction study, Journal of neural transmission 123 (2016) 895-904.

[25] K. Niitsu, M. J. Rice, J. F. Houfek, S. F. Stoltenberg, K. A. Kupzyk, C. R. Barron, A systematic review of genetic influence on psychological resilience, Biological research for nursing 21 (2019) 61-71.

[26] M. L. Sturge-Apple, D. Cicchetti, P. T. Davies, J. H. Suor, Differential susceptibility in spillover between interparental conflict and maternal parenting practices: Evidence for oxtr and 5-htt genes., Journal of Family Psychology 26 (2012) 431.

[27] S. Wüst, R. Kumsta, J. Treutlein, J. Frank, S. Entringer, T. G. Schulze, M. Rietschel, Sex-specific association between the 5-htt gene-linked polymorphic region and basal cortisol secretion, Psychoneuroendocrinology 34 (2009) 972-982.

[28] A. Fandiño-Losada, Y. Wei, E. Åberg, L. K. Sjöholm, C. Lavebratt, Y. Forsell, Influence of serotonin transporter promoter variation on the effects of separation from parent/partner on depression., Journal of affective disorders 144 (2013) 216-224.

[29] H. Steiger, J. Richardson, R. Joober, L. Gauvin, M. Israel, K. R. Bruce, N. N. Y. Kin, H. Howard, S. N. Young, The 5httlpr polymorphism, 
prior maltreatment and dramatic-erratic personality manifestations in women with bulimic syndromes, Journal of psychiatry \& neuroscience: JPN 32 (2007) 354.

[30] A. Caspi, K. Sugden, T. E. Moffitt, A. Taylor, I. W. Craig, H. Harrington, J. McClay, J. Mill, J. Martin, A. Braithwaite, et al., Influence of life stress on depression: moderation by a polymorphism in the 5-htt gene, Science 301 (2003) 386-389.

[31] J. Belsky, M. J. Bakermans-Kranenburg, M. H. Van IJzendoorn, For better and for worse: Differential susceptibility to environmental influences, Current directions in psychological science 16 (2007) 300-304.

[32] M. J. Bakermans-Kranenburg, M. H. van IJzendoorn, Oxytocin receptor (oxtr) and serotonin transporter (5-htt) genes associated with observed parenting, Social cognitive and affective neuroscience 3 (2008) 128-134.

[33] G. W. Brown, T. O. Harris, Depression and the serotonin transporter 5-httlpr polymorphism: a review and a hypothesis concerning geneenvironment interaction, Journal of affective disorders 111 (2008) 1-12.

[34] G. Parker, H. Tupling, L. B. Brown, A parental bonding instrument, British journal of medical psychology 52 (1979) 1-10.

[35] G. Parker, The parental bonding instrument: psychometric properties reviewed., Psychiatric developments 7 (1989) 317-335.

[36] G. Parker, The parental bonding instrument: a decade of research., Social Psychiatry and Psychiatric Epidemiology: The International Journal for Research in Social and Genetic Epidemiology and Mental Health Services (1990).

[37] A. Bonassi, T. Ghilardi, A. Truzzi, I. Cataldo, A. Azhari, P. Setoh, K. Shinohara, G. Esposito, Dataset on genetic and physiological adults' responses to social distress, Data in brief 13 (2017) 742-748.

[38] A. Bonassi, I. Cataldo, G. Gabrieli, J. N. Foo, B. Lepri, G. Esposito, Oxytocin receptor gene polymorphisms and early parental bonding interact in shaping instagram social behavior, International journal of environmental research and public health 17 (2020) 7232. 
[39] B. M. Trifiro, J. Gerson, Social media usage patterns: research note regarding the lack of universal validated measures for active and passive use, Social Media+ Society 5 (2019) 2056305119848743.

[40] A. G. Reece, C. M. Danforth, Instagram photos reveal predictive markers of depression, EPJ Data Science 6 (2017) 15.

[41] J. Y. Jang, K. Han, P. C. Shih, D. Lee, Generation like: comparative characteristics in instagram, in: Proceedings of the 33rd Annual ACM Conference on Human Factors in Computing Systems, ACM, pp. 40394042.

[42] I. E. Thorisdottir, R. Sigurvinsdottir, B. B. Asgeirsdottir, J. P. Allegrante, I. D. Sigfusdottir, Active and passive social media use and symptoms of anxiety and depressed mood among icelandic adolescents, Cyberpsychology, Behavior, and Social Networking 22 (2019) 535-542.

[43] T. M. Dumas, M. Maxwell-Smith, J. P. Davis, P. A. Giulietti, Lying or longing for likes? narcissism, peer belonging, loneliness and normative versus deceptive like-seeking on instagram in emerging adulthood, Computers in Human Behavior 71 (2017) 1-10.

[44] I. Ben-Gal, Outlier detection, in: Data mining and knowledge discovery handbook, Springer, 2005, pp. 131-146.

[45] F. Faul, E. Erdfelder, A.-G. Lang, A. Buchner, G* power 3: A flexible statistical power analysis program for the social, behavioral, and biomedical sciences, Behavior research methods 39 (2007) 175-191.

[46] J. L. Ivorra, J. Sanjuan, M. Jover, J. M. Carot, R. de Frutos, M. D. Molto, Gene-environment interaction of child temperament, Journal of Developmental \& Behavioral Pediatrics 31 (2010) 545-554.

[47] V. Mileva-Seitz, J. Kennedy, L. Atkinson, M. Steiner, R. Levitan, S. G. Matthews, M. J. Meaney, M. B. Sokolowski, A. S. Fleming, Serotonin transporter allelic variation in mothers predicts maternal sensitivity, behavior and attitudes toward 6-month-old infants, Genes, Brain and Behavior 10 (2011) 325-333. 
[48] D. Kiser, B. SteemerS, I. Branchi, J. R. Homberg, The reciprocal interaction between serotonin and social behaviour, Neuroscience \& Biobehavioral Reviews 36 (2012) 786-798.

[49] J. Belsky, C. Jonassaint, M. Pluess, M. Stanton, B. Brummett, R. Williams, Vulnerability genes or plasticity genes?, Molecular psychiatry 14 (2009) 746-754.

[50] C. Blaya, G. A. Salum, P. Moorjani, A. C. Seganfredo, E. Heldt, S. Leistner-Segal, J. W. Smoller, G. G. Manfro, Panic disorder and serotonergic genes (slc6a4, htr1a and htr2a): association and interaction with childhood trauma and parenting, Neuroscience letters 485 (2010) 11-15.

[51] V. Flasbeck, D. Moser, J. Pakusch, R. Kumsta, M. Brüne, The association between childhood maltreatment and empathic perspective taking is moderated by the 5-htt linked polymorphic region: Another example of "differential susceptibility", PloS one 14 (2019) e0226737.

[52] M. Wang, X. Tian, W. Zhang, Interactions between the combined genotypes of 5-httlpr and bdnf val66met polymorphisms and parenting on adolescent depressive symptoms: A three-year longitudinal study, Journal of Affective Disorders 265 (2020) 104-111.

[53] G. Miller, G. Zhu, M. J. Wright, N. K. Hansell, N. G. Martin, The heritability and genetic correlates of mobile phone use: A twin study of consumer behavior, Twin research and human genetics 15 (2012) 97-106.

[54] D. Deryakulu, Ö. F. Ursavaş, Genetic and environmental influences on problematic internet use: A twin study, Computers in Human Behavior 39 (2014) 331-338.

[55] C. York, A regression approach to testing genetic influence on communication behavior: Social media use as an example, Computers in Human Behavior 73 (2017) 100-109.

[56] A. Bonassi, I. Cataldo, G. Giulio, J. N. Foo, B. Lepri, E. Gianluca, P. 677 serotonin transporter gene polymorphisms and confidence in adult relationships affect the number of followed people on instagram, in: 33rd ECNP Congress-Posters, volume 40, pp. S385-S386. 
[57] L. E. Sherman, P. M. Greenfield, L. M. Hernandez, M. Dapretto, Peer influence via instagram: effects on brain and behavior in adolescence and young adulthood, Child development 89 (2018) 37-47.

[58] T. M. Dumas, M. A. Maxwell-Smith, P. F. Tremblay, D. M. Litt, W. Ellis, Gaining likes, but at what cost? longitudinal relations between young adults' deceptive like-seeking on instagram, peer belonging and self-esteem, Computers in Human Behavior (2020) 106467.

[59] L. E. Sherman, A. A. Payton, L. M. Hernandez, P. M. Greenfield, M. Dapretto, The power of the like in adolescence: effects of peer influence on neural and behavioral responses to social media, Psychological science 27 (2016) 1027-1035.

[60] H. Fisher, Lust, attraction, attachment: Biology and evolution of the three primary emotion systems for mating, reproduction, and parenting, Journal of Sex Education and Therapy 25 (2000) 96-104.

[61] S. F. Stoltenberg, C. C. Christ, G. Carlo, Afraid to help: social anxiety partially mediates the association between 5-httlpr triallelic genotype and prosocial behavior, Social neuroscience 8 (2013) 400-406.

[62] P. Sheldon, K. Bryant, Instagram: Motives for its use and relationship to narcissism and contextual age, Computers in human Behavior 58 (2016) 89-97.

[63] I. Sen, A. Aggarwal, S. Mian, S. Singh, P. Kumaraguru, A. Datta, Worth its weight in likes: Towards detecting fake likes on instagram, in: Proceedings of the 10th ACM Conference on Web Science, pp. 205-209.

[64] K. Zarei, R. Farahbakhsh, N. Crespi, Typification of impersonated accounts on instagram, in: 2019 IEEE 38th International Performance Computing and Communications Conference (IPCCC), IEEE, pp. 1-6. 